Proceedings

\title{
Investigation of the Relation between Follicular Atresia and Granulosa Cells in Terms of Cell Death Mechanisms in Premature Ovarian Failure Model ${ }^{+}$
}

\author{
Damla Akogullari 1,*, Elgin Turkoz Uluer ${ }^{2}$ and H. Seda Vatansever 1,3 \\ 1 Department of Histology \& Embryology, Institute of Medical Sciences, Manisa Celal Bayar University, \\ Manisa 45030, Turkey; sedavatansever@yahoo.com \\ 2 Department of Histology \& Embryology, Faculty of Medicine, Manisa Celal Bayar University, \\ Manisa 45030, Turkey; drelginturkoz@gmail.com \\ 3 Experimental Health Sciences Research Center, Near East University, Nicosia 99138, Cyprus \\ * Correspondence: daakogullari@gmail.com \\ † Presented at the 2nd International Cell Death Research Congress, Izmir, Turkey, 1-4 November 2018. \\ Published: 6 December 2018
}

\begin{abstract}
Premature Ovarian Failure; is characterized by the dysfunction or early depletion of ovarian reserves due to follicular loss in the ovary in women under age of 40. POF is the important cause of infertility and its etiology is still not clearly understood. Investigation of cell death mechanisms (CDM) that play a role in the follicular atresia (FA) triggered by excessive loss of granulosa cells (GCs) that provide metabolic support for oocyte and follicle development in the ovary will help to understand POF etiology. It was known that apoptosis and autophagy play a role in FA. Recent studies have shown that paraptosis, associated with endoplasmic reticulum stress (ERS), also exist in FA. POF model was established in C57BL/6 female mice by CTX and it was confirmed by increased follicle stimulating hormone (FSH), luteal hormone (LH) and decreased estradiol (E2) blood levels and follicle count. According to the results of the immunohistochemistry (IHC) cell death markers were significantly more expressed than control (C) and sham (S) groups in the POF model. In addition, more apoptotic cells were observed in the POF group compared to $\mathrm{C}$ and $\mathrm{S}$ in the TUNEL analysis. In consequence of this study apoptosis and autophagy as well as paraptosis play a role in the FA leading to POF, will help to develop new treatment protocols.
\end{abstract}

Keywords: Premature Ovarian Failure; cyclophosphamide; follicular atresia; apoptosis; autophagy; paraptosis

\section{Introduction}

Premature Ovarian Failure (POF) is characterized by the dysfunction or early depletion of ovarian reserve before the age of 40 , influences approximately $1 \%$ of in women in the worldwide, its etiology is still not clear. Loss of GCs with FA leads to premature depletion of ovarian reserves and POF formation. According to literature POF animal model is established with CTX [1-3]. The two most well-known cell death types in the FA process are apoptosis and autophagy. In addition, recently it has been suggested that paraptosis, which is a type of cell death has associated with ERS has been played a role in this process [1,4]. In this study, it was aimed to establish the POF model in C57BL/6 female mice that was induced by CTX and evaluation of apoptosis, autophagy and paraptosis via indirect IHC method in order to determine the CDM that play a role in FA process. 


\section{Material and Methods}

\subsection{Establish of the POF Animal Model}

Eighteen female C57BL/6 mice at the age of- 8 weeks randomly divided into 3 groups as $\mathrm{C}, \mathrm{S}$ and POF (n:6, for each group). POF model has been established with CTX (sc-202117, Chem Cruz, Dallas, TX, USA) treatment. No treatment was applied to the $C$ group. The $S$ group were given an equal amount of saline. CTX was administered intraperitoneally one every 2 days for 3 weeks; the first dose was $70 \mathrm{mg} / \mathrm{kg}$, the ongoing doses were $30 \mathrm{mg} / \mathrm{kg}$. At the end of 3 weeks, $3 \mathrm{~mL}$ of blood was taken intracardiac under general anesthesia for analysis of hormone serum levels and then animals sacrificed by cervical dislocation and ovaries were taken.

\subsection{Hormon Assay}

The serum concentration level of FSH, LH and E2 were measured by ELISA kit (SHANGHAI YEHUA, Shanghai, China) as the indicators to assess sexual hormone level in animal models.

\subsection{Histopathological Examination}

For histopathological examination, all ovary tissues were fixed for $48 \mathrm{~h}$ in $10 \%$ formaldehyde at room temperature. Samples were embedded in paraffin after routine histological embedding procedures, $5 \mu \mathrm{m}$ serial sections were cut from the paraffin blocks with the rotary microtome. From the first section, routine H\&E staining was performed in each of the five sections for every groups. The follicle classification [1] and counting was performed under a light microscope.

\subsection{Immunohistochemical Staining}

For the IHC analysis, paraffin embedded sections were de-paraffinized. Among the markers of cell death mechanisms, IHC staining was performed with anti-caspase 3 (sc-56053, Santa Cruz, Texas, Tex, USA), anti-caspase 8 (sc-70501, Santa Cruz, TX, USA) and anti-caspase 9 (sc-56076, Santa Cruz, Texas, Tex, USA) for apoptosis, anti-Lamp1 (sc-20011, Santa Cruz, TX, USA) and anti-MAP LC3 $\beta$ (sc271625, Santa Cruz, TX, USA) for autophagy, and anti-GRP78 (sc-376768, Santa Cruz, Texas, Tex, USA) and anti-CHOP (Abp50978, Abbkine, Wuhan, China) primer antibodies for paraptosis. The terminal deoxynucleotidyl transferase (TdT)-mediated deoxyuridine triphosphate (dUTP) nick end labeling (TUNEL-Millipore, S7101, Temecula, CA, USA) assay was performed according to the manufacturer's instructions. Data analyses included calculations of group means and standard deviation (SD). Data were analyzed by Tukey-Kramer Multiple Comparison Test using one way ANOVA with significance set at $p$ value $<0.05$.

\section{Results}

\subsection{Biochemical Results}

A decrease in E2 level was observed when the serum FSH and LH levels were increased in the POF group according to the control and sham group (Table 1).

Table 1. Serum concentration level of FSH, LH and E2 of C, S and POF groups (Mean \pm SD).

\begin{tabular}{cccc}
\hline & FSH & LH & E2 \\
\hline CONTROL & $6.79 \pm 1.73$ & $3.93 \pm 1.19$ & $119.05 \pm 19.63$ \\
SHAM & $8.89 \pm 1.99$ & $4.89 \pm 3.72$ & $112.85 \pm 48.24$ \\
POF & $10.98 \pm 3.45$ & $5.11 \pm 1.51$ & $107.93 \pm 27.22$ \\
\hline
\end{tabular}




\subsection{Histophalogical Results}

H\&E Staning and Follicle Counting

As a result of immunohistochemical staining; caspase-3, caspase-8, and caspase-9 immunoreactivities were mild in the control and sham groups, caspase- 3 immunoreactivity was mild to moderate in the POF group, caspase- 8 and caspase- 9 immunoreactivities were moderate; Lamp1 and MAP LC3 $\beta$ immunoreactivities were mild in the control and the sham groups, while in the POF group, Lamp1 immunoreactivity was mild to moderate and MAP LC3 $\beta$ immunoreactivity was moderate; CHOP and GRP78 immunoreactivities were observed to be moderate in the control and sham group and moderate in the POF group.

\subsection{Immunohistochemical Staining}

As a result of immunohistochemical staining; caspase-3, caspase-8, and caspase-9 immunoreactivities were mild in the control and sham groups, caspase- 3 immunoreactivity was mild to moderate in the POF group, caspase- 8 and caspase- 9 immunoreactivities were moderate; Lamp1 and MAP LC3 $\beta$ immunoreactivities were mild in the control and the sham groups, while in the POF group, Lamp1 immunoreactivity was mild to moderate and MAP LC3 $\beta$ immunoreactivity was moderate; CHOP and GRP78 immunoreactivities were observed to be moderate in the control and sham group and moderate in the POF group (Figure 1).
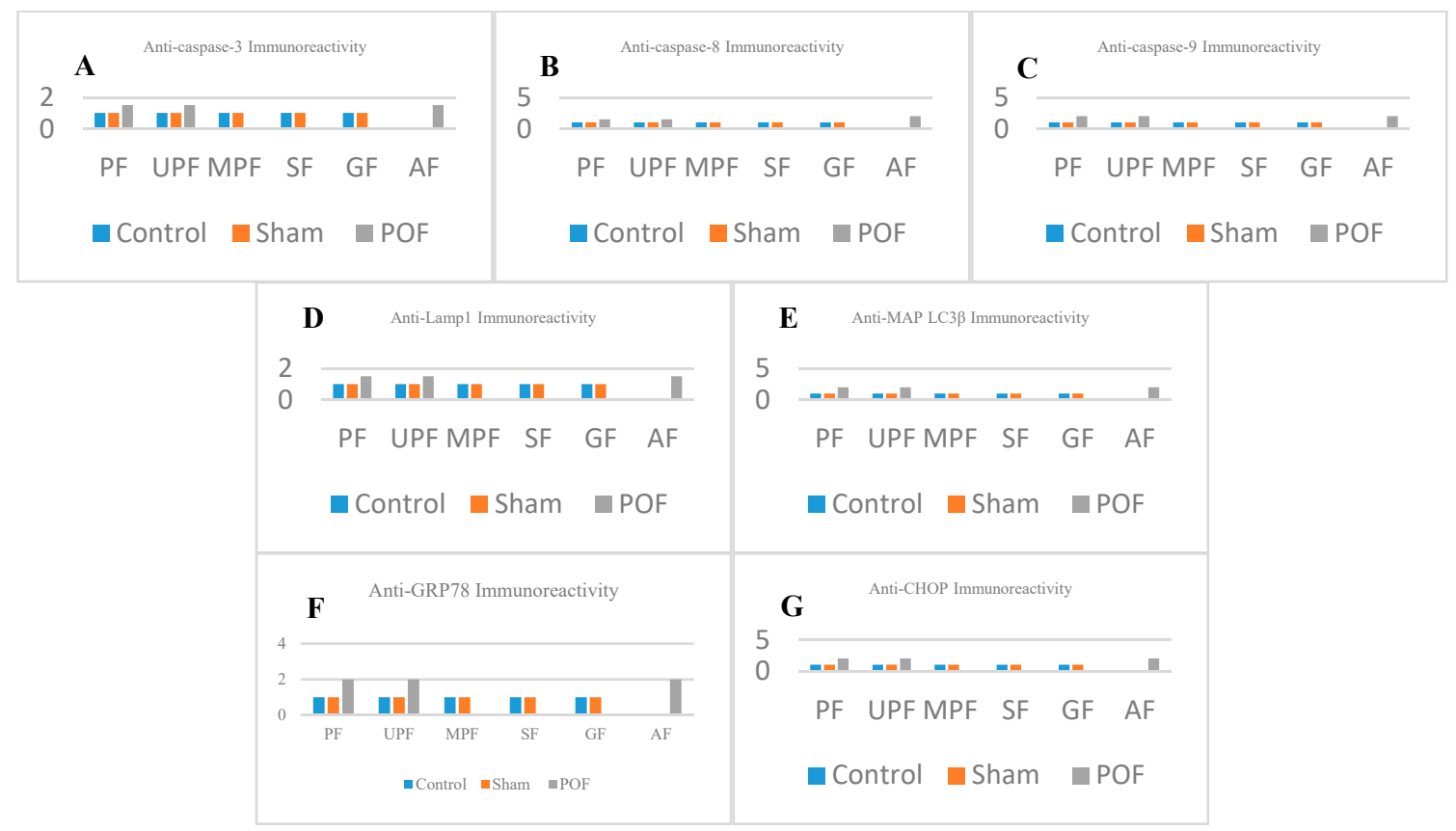

Figure 1. IHC analysis result of CDMs markers for apoptosis; (A) caspase 3, (B) caspase 8 and (C) caspase 9, for autophagy; (D) Lamp1, (E) MAP LC3 $\beta$ and for paraptosis; (F) GRP78, (G) CHOP primer antiboies's immunoreactivities. PF: Primordial Follicle, UPF: Unilaminar Primary Follicle, MPF:Multilaminar Primary Follicle, SF: Seconder Follicle, GF: Graaffian Follicle, AF: Atresic Follicle.

\subsection{TUNEL Assay}

As a result of TUNEL analysis, more apoptotic cells were detected in the POF group than in the $\mathrm{C}$ and $\mathrm{S}$ group in the ovary sections (Figure 2).

\begin{tabular}{|c|c|c|}
\hline CONTROL & SHAM & POF \\
\hline & C & \\
\hline & & \\
\hline
\end{tabular}

Figure 2. Representative images of TUNEL analysis results for all groups (Scale bar: $10 \mu \mathrm{m}$ ). 


\section{Discussion and Conclusions}

In the present study compatible with the literature, a mouse POF model was successfully established by CTX. In the studies about POF model reported that FSH and LH serum hormon levels increase and E2 levels decrease in the POF groups comparison with the $C$ groups [1-3]. It is known that POF is a disease associated with follicule depletion and GC death in FA process. In studies about $\mathrm{POF}$, although the $\mathrm{C}$ groups have all follicule of type, amoung the POF groups have no mature follicules but many atretic follicle in the ovaries [1,3]. As a result of follicle count almost all of the follicle types were observed in the $C$ and the $S$ groups while in the POF group atretic follicles were significantly increased and no mature follicles were observed in our study. To clarify the etiology of POF, the FA process needs to be well investigated. In addition to apoptosis and autophagy which play a role in the process of FA, studies have also shown that paraptosis, a type of cell death associated with ERS, has recently been involved [1,4]. In our study, apoptosis and autophagy markers, involved in the FA process, was significantly increased in POF group compared to $C$ and $S$ groups. Moreover, the presence of paraptosis in the FA process was confirmed by a significant increase in paraptosis's markers compared to the $C$ and the $S$ groups. These findings will help to develop new treatment protocols for POF by providing a better understanding of the CDMs involved in the etiology of POF.

Author Contributions: E.T.U. and H.S.V. conceived, designed the experiments and analyzed the data; D.A. performed the experiments; E.T.U. and D.A. wrote the paper.

Acknowledgments: This study was supported by Manisa Celal Bayar University Scientific Research Projects Unit with the project numbered 2017-149.

Conflicts of Interest: The authors declare no conflict of interest.

\section{References}

1. Yan, Z.; Dai, Y.; Fu, H.; Zheng, Y.; Bao, D.; Yin, Y.; Chen, Q.; Nie, X.; Hao, Q.; Hou, D.; Cui, Y. Curcumin Exerts a Protective Effect against Premature Ovarian Failure in Mice. J. Mol. Endocrinol. 2018, 60, 261-271, doi:10.1530/JME-17-0214. Available online: https://www.ncbi.nlm.nih.gov/pubmed/29437881 (accessed on 16 October 2018).

2. Zhang, T.; Yan, D.; Yang, Y.; Ma, A.; Li, L.; Wang, Z.; Pan, Q.; Sun, Z. The Comparison of Animal Models for Premature Ovarian Failure Established by Several Different Source of Inducers. Regul. Toxicol. Pharmacol. 2016, 81, 223-232, doi:10.1016/j.yrtph.2016.09.002. Available online: https://www.ncbi.nlm.nih.gov/pubmed/27612992 (accessed on 16 October 2018).

3. Xiong, Y.; Liu, T.; Wang, S.; Chi, H.; Chen, C.; Zheng, J. Cyclophosphamide Promotes the Proliferation Inhibition of Mouse Ovarian Granulosa cells and Premature Ovarian Failure by Activating the IncRNAMeg3-p53-p66Shc Pathway. Gene 2017, 5, 1-8, doi:10.1016/j.gene.2016.10.011. Available online: https://www.ncbi.nlm.nih.gov/pubmed/27729272 (accessed on 16 October 2018).

4. Torres-Ramírez, N.; Escobar, M.L.; Vázquez-Nin, G.H.; Ortiz, R.; Echeverría, O.M. Paraptosis-Like Cell Death in Wistar Rat Granulosa Cells. Dev. Growth Differ. 2016, 58, 651-663, doi:10.1111/dgd.12322. Available online: https://www.ncbi.nlm.nih.gov/pubmed/27684714 (accessed on 16 October 2018).

(C) 2018 by the authors. Licensee MDPI, Basel, Switzerland. This article is an open access article distributed under the terms and conditions of the Creative Commons Attribution (CC BY) license (http://creativecommons.org/licenses/by/4.0/). 